\title{
Method for Texture Mapping of In-Vehicle Camera Image in 3D Map Creation
}

\author{
Kohei Arai $^{1}$ \\ Department of Information Science, Saga University, Saga City, Japan
}

\begin{abstract}
Method for texture mapping of in-vehicle camera image in 3D map creation is proposed. Top view of ground cover targets can be mapped easily. For instance, aerial photos, high spatial resolution of satellite imagery data allows creation of top view of ground cover targets and also map creation. It can be used for pedestrian navigations. On the other hand, side view of ground cover targets is not so easy to obtain. In this paper, two methods are proposed. One is to use acquired photos with cameras mounted on the dedicated cars. The other one is to use high spatial resolution of satellite imagery data, such as IKONOS, Orbview, etc. Through experiments with the aforementioned two methods, it is found that texture mapping for the ground cover targets can be done with the two proposed methods in an efficient manner.
\end{abstract}

Keywords-Texture mapping; high spatial resolution of satellite imagery data; 3D map

\section{INTRODUCTION}

It is aimed at visually representing a three-dimensional map easy to understand for people who cannot read a twodimensional map. "How fast can you map?" is the question. In particular for the texture mapping 1 of the side wall of the buildings, the followings are problems,

- The number of buildings is innumerable.

- It cannot spend time on one building.

Therefore, the followings are getting much important,

Create a tool and make it workable.

(Win32 $\mathrm{API}^{2}, \mathrm{OpenCV}^{3}$, OpenGL ${ }^{4}$ ) These are candidates for the texture mapping. In this connection, there are the following three questions,

1) How to take a picture?

- Because the way of shooting varies depending on the person, it is diverse, so that the following conditions have to be set in this research.

- Shoot directly in front.

- Take it from an angle.

(It is assumed that 4 corners of the building are contained in one picture)

\footnotetext{
${ }^{1}$ https://en.wikipedia.org/wiki/Texture_mapping

${ }^{2}$ https://ja.wikipedia.org/wiki/Windows_API

${ }^{3}$ https://opencv.org/

${ }^{4}$ https://ja.wikipedia.org/wiki/OpenGL
}

2) How to map the acquired pictures:

- Photos taken from just in front are easy to map.

- If a picture is taken from an angle, rotate it about the height direction as if it were to be taken from the front as if it were taken. Therefore, three dimensional information required.

3) Mapping models:

Extract three-dimensional information from multiple pictures. Then, Affine Approximate Projection Model $^{5}$ can be used with the following model,

\section{(Representative: Para perspective Projection Model $^{6}$ )}

Factorization method $^{7}$

Pedestrian navigation using three-dimensional map

It became easy to understand its position and the direction heading to the destination. Issues in $3 \mathrm{D}$ map creation is the followings,

One year to create three-dimensional map around the major 5300 intersections of government-designated cities in Japan. Therefore, 3D map has to be created in an efficient manner considering the followings.

Difficulty in collecting images around the intersection

It takes time to capture, map the image to a 3-dimensional map.

In this paper, two methods are proposed. One is to use acquired photos with cameras mounted on the dedicated cars. The other one is to use high spatial resolution of satellite imagery data, such as IKONOS $^{8}$, Orbview ${ }^{9}$, etc. Through experiments based on the aforementioned two methods, it is found that texture mapping for the ground cover targets can be done with the two proposed methods in an efficient manner.

The next section describes research background and the proposed method. Then, some experiments are described followed by conclusion with some discussions. After that some future research works are described.

\footnotetext{
${ }_{6}^{5} \mathrm{http} / / /$ www.isprs.org/proceedings/XXXVII/congress/4_pdf/231.pdf
}

https://www.cse.unr.edu/ bebis/CS791E/Notes/PerspectiveProjection.pdf

${ }^{7}$ https://en.wikipedia.org/wiki/Factorization

${ }^{8} \mathrm{https}$ ///www.satimagingcorp.com/satellite-sensors/ikonos/

${ }^{9}$ https://lta.cr.usgs.gov/satellite_orbview3 


\section{RESEARCH BACKGROUND AND THE PROPOSED METHOD}

\section{A. Research Background and the Related Research}

Mashups by orchestration and widget-based personal environments: key challenges, solution strategies, and an application is proposed [1]. Meanwhile, semantic mashups. intelligent reuse of Web resources is well described [2]. On the other hand, "What is Web 2.0. Design Patterns and Business Models for the Next Generation of Software" is well reported [3]. Also, "Services Mashups: The New Generation of Web Applications" is proposed [4]. In the meantime, "As Bing Takes Over Yahoo Search, SearchMonkey Dies, BOSS Is No Longer Free, But Site Explorer Still Works". is well reported [5].

Mashup based content search engine for mobile devices is proposed and introduced [6] together with module based content adaptation of composite e-learning content for delivering to mobile learners [7]. Also, efficiency improvements of e-learning document search engine for mobile browser is proposed [8] while efficiency improvement of elearning document search engine for mobile browser is discussed [9]. Video searching optimization with supplemental semantic keyword for e-learning video searching, is proposed [10] already. 3D Interactive visualization for inter-cell dependencies of spreadsheets is proposed [11] while "IANA Considerations" of the application/json media type for JavaScript Object Notation (JSON) is well reported [12].

Meanwhile, e-learning system utilizing learners' characteristics recognized through learning process with Open Simulator is proposed [13] together with avatar utilized Q/A system of e-learning content designed with Open Simulator [14]. These are related research works to the proposed Method for texture mapping of in-vehicle camera image in 3D map creation. Hvidsten, Mike [15] introducing the OpenGL texture mapping guide in his homepages.gac.edu. (Retrieved $22 \mathrm{March}$ 2018).

\section{B. Conventional Method}

Take GoogleSketchUp ${ }^{10}$ of 3D software provided by Google for free. Using this, texture mapping can be done. This is a matter of the number of processes and the length of processing time.

Firstly, side view of photo of the building in concern is acquired. Four corners of the building can be extracted easily as shown in Fig. 1. Then, mapped it using the photograph from the front (no rotation). Thus, the side view of the building wall can be mapped with texture.

\section{Proposed Method}

As described in the previous section, one of the serious problems of the conventional method is the number of processes and too long processing time. In the proposed method, at least two photos are acquired in advance to the texture mapping process. Namely, manually select the four corners of the building taken in the photograph, apply projective transformation as a feature point, and map to the

\footnotetext{
${ }^{10}$ https://www.sketchup.com/, https://googlesketchup.jp.uptodown.com/windows
}

side. This simulation is repeated for five times to measure the average required time. The basic process flow of the proposed texture mapping method is illustrated in Fig. 2.

There are two different image sources, car mounted camera and high spatial resolution camera or radiometer onboard satellite. From the car mounted camera, side view of texture from an aspect of image is acquired while top view of texture and a small portion of side view texture image are acquired from the high spatial resolution radiometer onboard satellite. Occluded side view texture can be assumed from the portion of satellite image. Fig. 2 shows, also, the relation among the coordinate systems of sun illumination, object building or houses, car mounted camera in the world coordinate system. Although the coordinate systems of sun illumination, and the object are fixed, the car mounted camera coordinate system can be moved and vibrated. By using acquired image by the camera, it is possible to estimate the movement as well as vibration in the world coordinate system. Meanwhile, the height of the object can be estimated with the satellite image as shown in the later section.

Fig. 3 shows the process flow of the proposed texture mapping method. Proposal on a method for efficient mapping using pictures taken from onboard camera

It is premised that 3-dimensional information of the building is acquired in advance by Zenrin Co., Ltd. ${ }^{11}$ etc. Then, ortho images (images after orthogonal projection conversion) can be created. Fig. 4 shows an example of the texture mapping processed image.

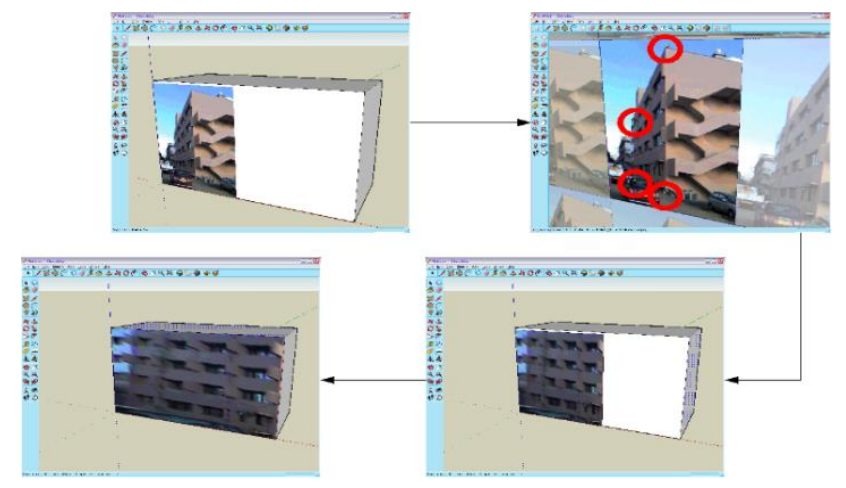

Fig. 1. Side View Texture Mapping with GoogleSketchUp.

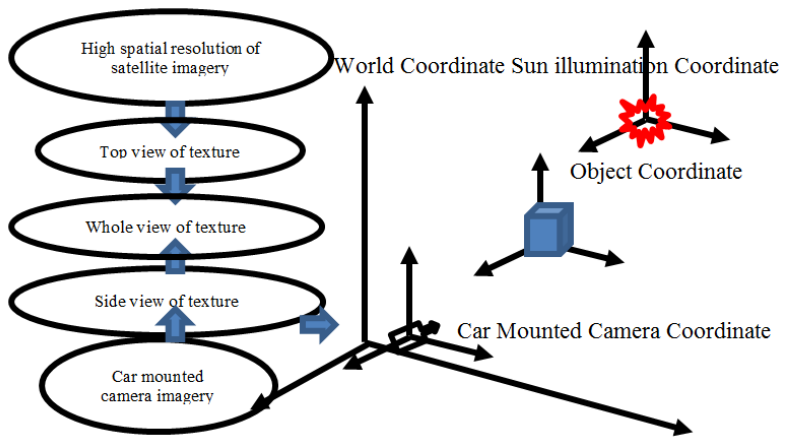

Fig. 2. Basic Process Flow of the Proposed Texture Mapping Method.

\footnotetext{
${ }^{11}$ https://www.zenrin.co.jp/english/index.html
} 


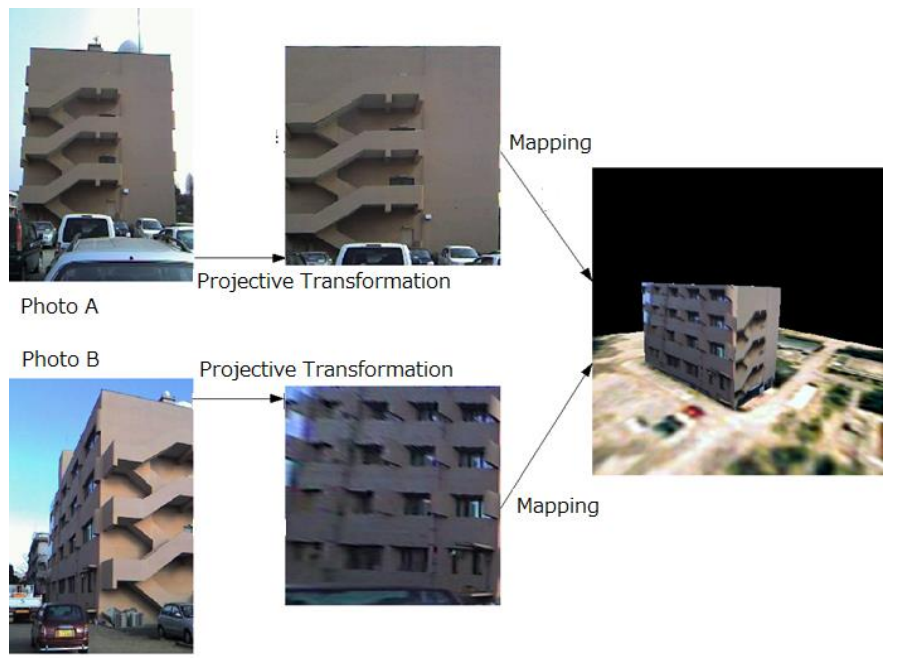

Fig. 3. Process Flow of the Proposed Texture Mapping Method.

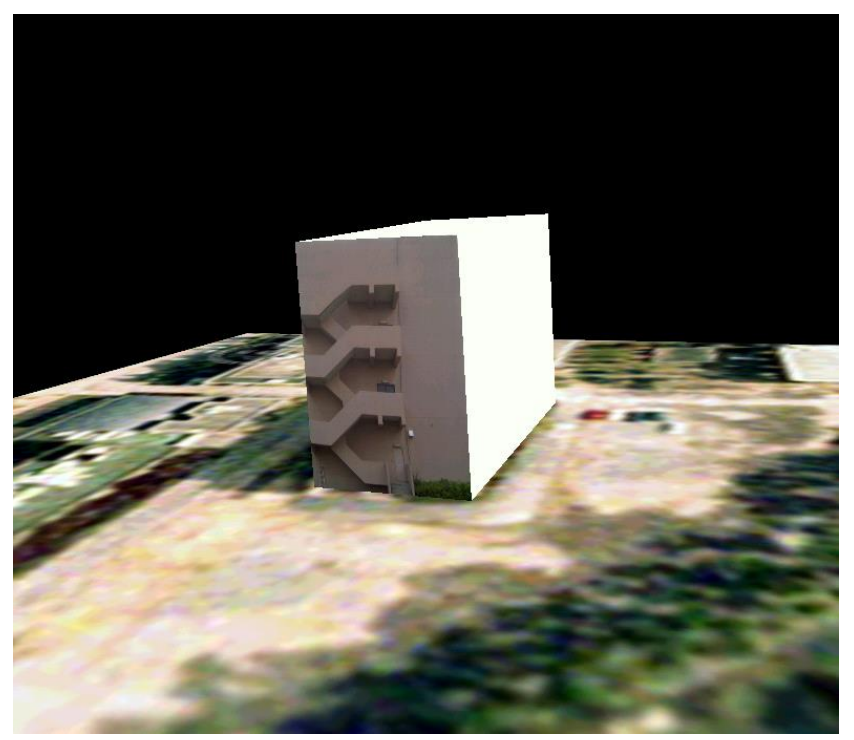

Fig. 4. Example of the Texture Mapping Processed Image.

\section{Texture Mapping with High Spatial Resolution of Satellite Images}

More than two of off-nadir view of high spatial resolution of satellite images are assumed to be acquired. From the images, the following processes are going to be done,

1) Estimation of 3-D building height

2) IKONOS image segmentation

3) Texture Mapping

Fig. 5 shows an example of high spatial resolution of IKONOS satellite images. IKONOS satellite sensor characteristics are as follows,

1) Launch Date: 24 September 1999 at Vandenberg Air Force Base, California, USA

2) Operational Life: Over 7 years
3) Orbit: 98.1 degree, sun synchronous

4) Speed on Orbit: 7.5 kilometers per second

5) Speed Over the Ground: 6.8 kilometers per second

6) Revolutions Around the Earth: 14.7, every 24 hours

7) Altitude: 681 kilometers

8) Resolution at Nadir: 0.82 meters panchromatic; 3.28 meters multispectral

9) Resolution $26^{\circ}$ Off-Nadir: 1.0 meter panchromatic; 4.0 meters multispectral

10)Image Swath: 11.3 kilometers at nadir; 13.8 kilometers at $26^{\circ}$ off-nadir

11)Equator Crossing Time: Nominally 10:30 AM solar time

12)Revisit Time: Approximately 3 days at $40^{\circ}$ latitude

13)Dynamic Range: 11-bits per pixel

14)Image Bands: Panchromatic, blue, green, red, near IR.

Estimate the height of the building using the shadow of the building from the image. If $\theta$ can be estimated, $\mathrm{h}$ ' is obtained as shown in Fig. 6.

Therefore, building height in concern can be estimated by using the calculated length of the shadow.

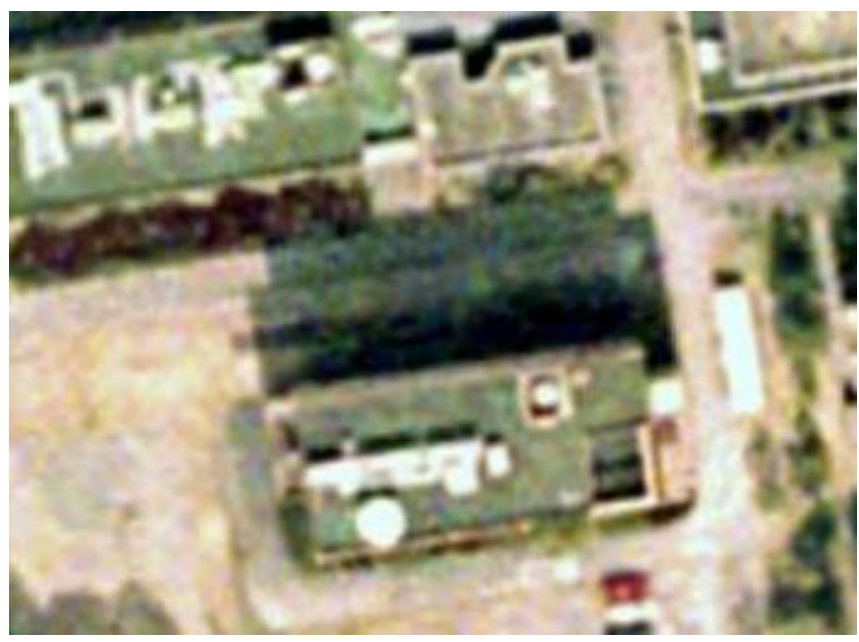

Fig. 5. Example of High Spatial Resolution of IKONOS Satellite Images.

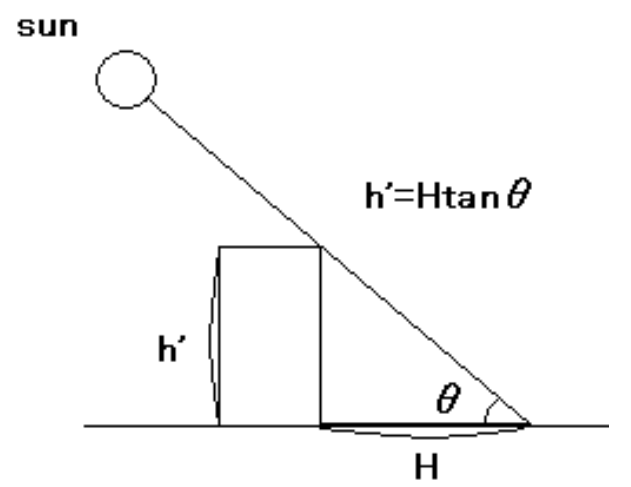

Fig. 6. Method for Estimation of Building Height in Concern. 


\section{EXPERIMENT}

\section{A. Preliminary Experiment}

Looking at the IKONOS image cut out for the texture, the difference in resolution appears depending on the place to use for the 3D map. It is necessary to correct pixels and create images to be used for texture. Due to the appearance problem, there is a possibility that the resolution has to be improved.

Fig. 7(a) and (b) show the one side of the extracted building derived from the IKONOS image and the other side of the extracted building derived from the IKONOS image, respectively.

As shown in Fig. 8, occluded side of the texture cannot be mapped obviously. That is way, at least two satellite images which are acquired from the different look angles are required.

Consider an efficient method of obtaining texture from a strabismus image. It also solves the problem of resolution when extracting IKONOS images.

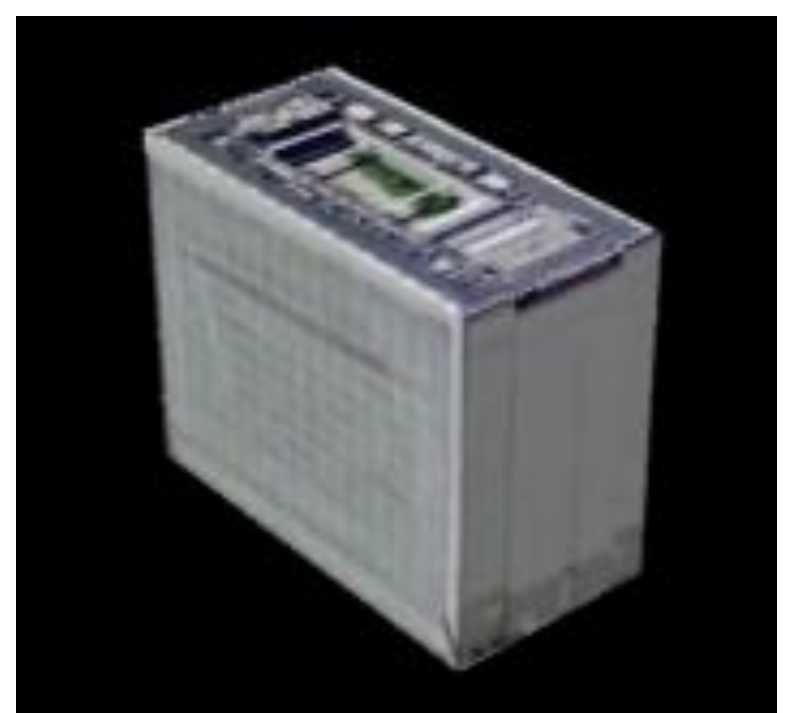

(a) One Side.

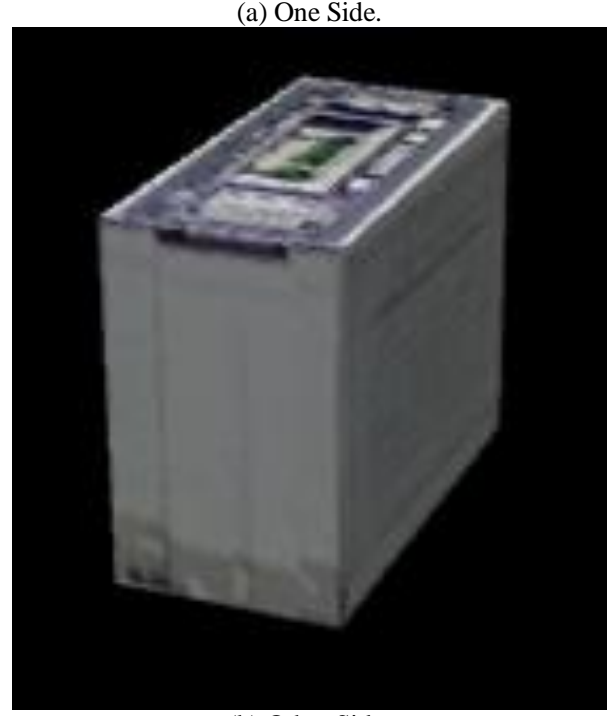

(b) Other Side.

Fig. 7. One Side and the other side of the Extracted Building Derived from the IKONOS Image.

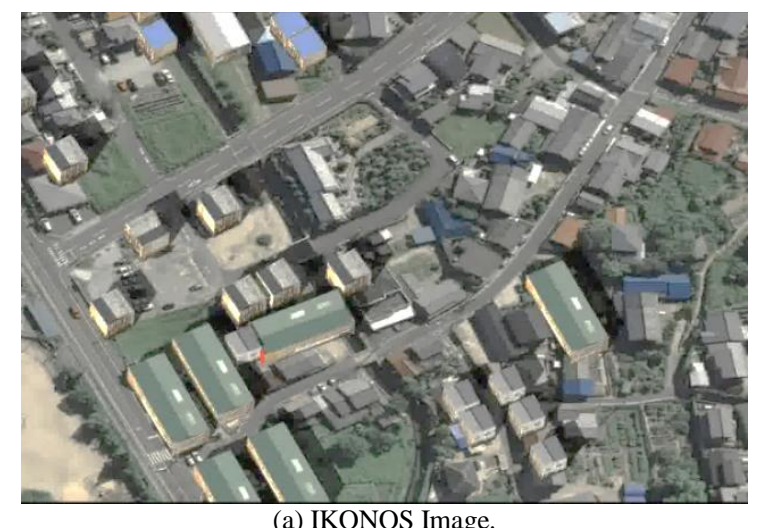

(a) IKONOS Image

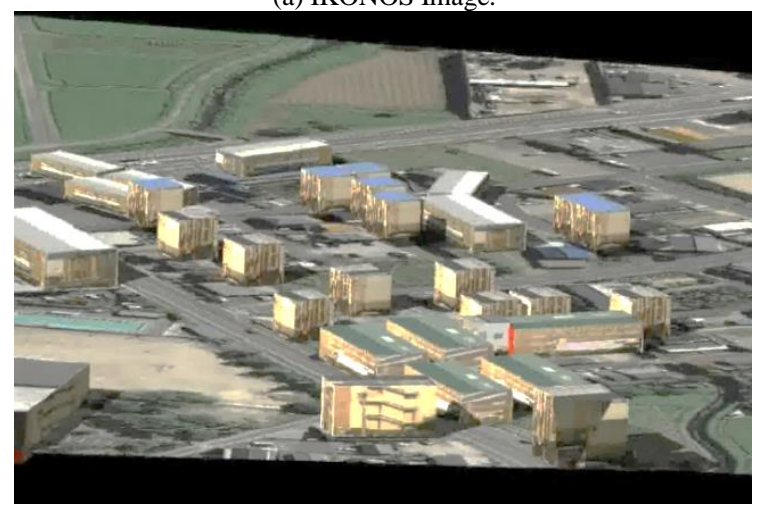

(b) Side View of the Building in Front cannot be seen

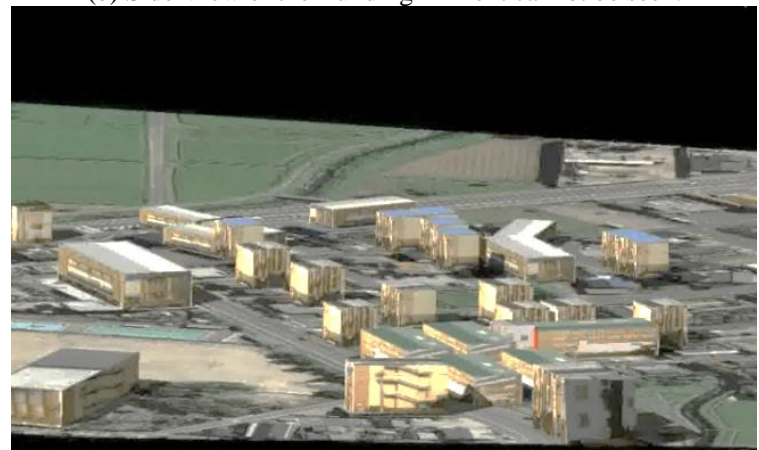

(c) Side View of the Building in Front can be seen with the Image Acquired with Automobile Mounted Camera.

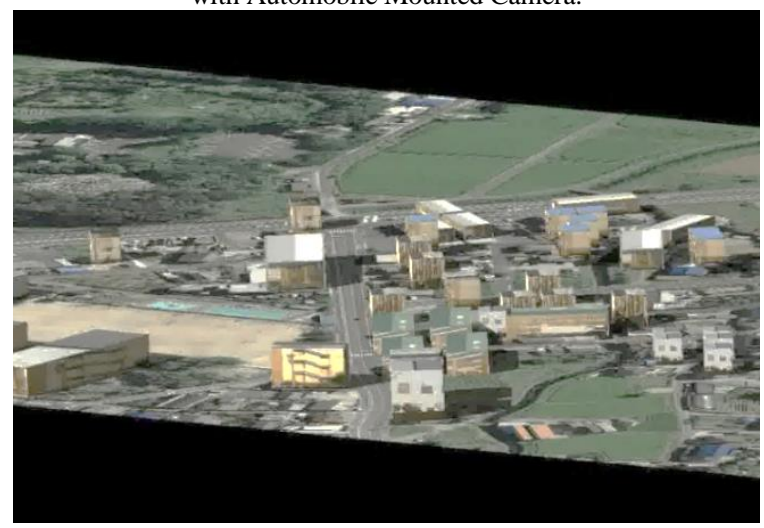

(d) Clear Side View Texture Mapping is Done for All the Ground Cover Target.

Fig. 8. Side View Texture Mapping with the Proposed Method.

\section{B. Effectiveness of the Proposed Method}

The proposed additional method for 3D map creation is to use both high spatial resolution of satellite imagery data and 
the cameras mounted on automobile. Although the proposed fundamental method for 3D map creation is to use high spatial resolution of satellite imagery data, it is not always to obtain more than two high spatial resolution of satellite imagery data from the different aspect. Therefore, if the more than two of high spatial resolution of satellite imagery data from the different aspect cannot be obtained and enough off-nadir view of high spatial resolution of satellite imagery data are not acquired, then the automobile mounted camera images are used only for the occluded ground cover targets for texture mapping.

Fig. 8 shows examples. Fig. 8(a) shows IKONOS image of Saga, Japan. Side wall of some of the houses and the building can be seen. It, however, is not for all the ground cover targets. Therefore, the side view of the building in front of Fig. 8(b) cannot be seen. Side view of the building in front can be seen with the image acquired with automobile mounted camera as shown in Fig. 8(c). It, however, can be done with the proposed method. Clear side view texture mapping is done for all the ground cover target as shown in Fig. 8(d).

\section{CONCLUSION}

Method for texture mapping of in-vehicle camera image in $3 \mathrm{D}$ map creation is proposed. Top view of ground cover targets can be mapped easily. For instance, aerial photos, highly spatial resolution of satellite imagery data allows creation of top view of ground cover targets and also map creation. It can be used for pedestrian navigations. On the other hand, side view of ground cover targets are not so easy to obtain. In this paper, two methods are proposed. One is to use acquired photos with cameras mounted on the dedicated cars. The other one is to use high spatial resolution of satellite imagery data, such as IKONOS, Orbview, etc. Through experiments with the aforementioned two methods, it is found that texture mapping for the ground cover targets can be done with the two proposed methods in an efficient manner.

The examples shows the effectiveness of the proposed method. In the IKONOS image of Saga, Japan., side wall of some of the houses and the building can be seen. It, however, is not for all the ground cover targets. Therefore, the side view of the building in front cannot be seen. Side view of the building in front can be seen with the image acquired with automobile mounted camera. It, however, can be done with the proposed method. Clear side view texture mapping is done for all the ground cover target.

\section{FUTURE RESEARCH}

Further study is required for further examples. The proposed method has to be attempted for more different target sites such as buildings are overlapped from the viewing angles, and so on. Also, it would better to apply the proposed method in the different environmental conditions, motion vibrations, night time observation, a bad weather conditions, and so on. Then limitation of the proposed method will be clarified.

\section{ACKNOWLEDGMENT}

The author would like to tank Mr. Kazunori Kinoshita, Mr. Tessei Yamaguchi of former students of the Saga University Japan for his effort to conduct the experiments.

\section{REFERENCES}

[1] Ahmet Soylu, Felix Mödritscher, Fridolin Wild, Patrick De Causmaecker, Piet Desmet.. "Mashups by Orchestration and Widgetbased Personal Environments: Key Challenges, Solution Strategies, and an Application." Program: Electronic Library and Information Systems 46 (4): 383-428. 2012.

[2] Endres-Niggemeyer, Brigitte ed.. Semantic Mashups. Intelligent Reuse of Web Resources. Springer. ISBN 978-3-642-36402-0 2013.

[3] O'Reilly, T., What is Web 2.0. Design Patterns and Business Models for the Next Generation of Software, p. 302005.

[4] Benslimane, D.; Dustdar, S.; Sheth, A.. "Services Mashups: The New Generation of Web Applications". IEEE Internet Computing 10 (5): $13-$ 15. doi:10.1109/MIC.2008.110 2008.

[5] Tuesday, August 17th,. "As Bing Takes Over Yahoo Search, SearchMonkey Dies, BOSS Is No Longer Free, But Site Explorer Still Works". TechCrunch. Retrieved 2012-09-18. 2010 (2010-08-17).

[6] Kohei Arai, Mash-up based content search engine for mobile devices, International Journal of Advanced Research in Artificial Intelligence, 2, 5, 39-43, 2013.

[7] Kohei Arai and Tolle Herman, Module based content adaptation of composite e-learning content for delivering to mobile learners, International Journal of Computer Theory and Engineering, 3, 3, 382387, 2011.

[8] Kohei Arai, Herman Tolle, Efficiency improvements of e-learning document search engine for mobile browser, International Journal of Research and Reviews on Computer Science, 2, 6, 1287-1291, 2011.

[9] K.Arai, T.Herman, Efficiency improvement of e-learning document search engine for mobile browser, International Journal of Research and review on Computer Science, 2, 6, 1287-1291, 2012.

[10] K.Arai, T.Herman, Video searching optimization with supplemental semantic keyword for e-learning video searching, International Journal of Research and Review on Computer Science, 3, 3, 1640-1644, 2012.

[11] H. Shiozawa, K. Okada, Y. Matsushita: 3D Interactive Visualization for Inter-Cell Dependencies of Spreadsheets, Proc. IEEE InfoVis '99, pp. 79-82, Oct. 1999.

[12] Douglas Crockford (July 2006). "IANA Considerations". The application/json Media Type for JavaScript Object Notation (JSON). IETF. sec. 6. RFC 4627. https://tools.ietf.org/html/rfc4627\#section-6. Retrieved October 21, 2009.

[13] Kohei Arai, Anik Nur Handayani, E-learning system utilizing learners' characteristics recognized through learning process with Open Simulator, International Journal of Advanced Research in Artificial Intelligence, 2, 4, 8-12, 2013.

[14] Kohei Arai, A.N.Hardiyani, Avatar utilized Q/A system of e-learning content designed with Open Simulator, Proceedings of the International Conference on Convergence Content 2012, 149-150, 2012.

[15] Hvidsten, Mike (Spring 2004). "The OpenGL Texture Mapping Guide". homepages.gac.edu. Retrieved 22 March 2018.

\section{AUTHOR's PROFILE}

Kohei Arai, He received BS, MS and PhD degrees in 1972, 1974 and 1982, respectively. He was with The Institute for Industrial Science and Technology of the University of Tokyo from April 1974 to December 1978 also was with National Space Development Agency of Japan from January, 1979 to March, 1990. During from 1985 to 1987, he was with Canada Centre for Remote Sensing as a Post Doctoral Fellow of National Science and Engineering Research Council of Canada. He moved to Saga University as a Professor in Department of Information Science on April 1990. He was a councilor for the Aeronautics and Space related to the Technology Committee of the Ministry of Science and Technology during from 1998 to 2000. He was a councilor of Saga University for 2002 and 2003. He also was an executive councilor for the Remote Sensing Society of Japan for 2003 to 2005. He is an Adjunct Professor of University of Arizona, USA since 1998. He also is Vice Chairman of the Science Commission "A" of ICSU/COSPAR since 2008 then he is now award committee member of ICSU/COSPAR. He wrote 37 books and published 570 journal papers. He received 30 of awards including ICSU/COSPAR Vikram Sarabhai Medal in 2016, and Science award of Ministry of Mister of Education of Japan in 2015. He is now Editor-in-Chief of IJACSA and IJISA. http://teagis.ip.is.saga-u.ac.jp/index.html 